\title{
Dynamics of power distribution in multimode semiconductor lasers with optical feedback
}

\author{
J.M. Buldú ${ }^{a}$, F. Rogister ${ }^{b}$, J. Trull ${ }^{a}$, C. Serrat ${ }^{a}$, M.C. Torrent ${ }^{a}$, \\ Claudio R. Mirasso ${ }^{c}$ and J. García-Ojalvo ${ }^{a}$ \\ ${ }^{a}$ Departament de Física i Enginyeria Nuclear, Universitat Politècnica de Catalunya, \\ Colom 11, E-08222 Terrassa, Spain \\ ${ }^{b}$ Advanced Research in Optics, Service d'Electromagnétisme et de Telécomunicacions, \\ Faculté Polytechnique de Mons, 31 Boulevard Dolez, B-7000 Mons, Belgium \\ ${ }^{c}$ Departament de Física, Universitat de les Illes Balears, E-07071 Palma de Mallorca, Spain
}

\begin{abstract}
Semiconductor lasers with optical feedback are prone to exhibit unstable behavior. When working near threshold with moderate to low optical feedback, intensity dropouts are observed. These intensity drops, also called lowfrequency fluctuations, occur both in single-mode and multimode semiconductor lasers. In this paper, the dynamics of the power distribution between the longitudinal modes of a multimode semiconductor laser is experimentally and numerically analyzed in the low-frequency fluctuation regime. It is observed that power dropouts of the total intensity, corresponding to drops in the dominant modes of the laser, are invariably accompanied by sudden activations of several longitudinal side modes. These activations are seen not to be simultaneous to the dropouts of the main modes, but to occur after them. The phenomenon is statistically analyzed in a systematic way, and the corresponding delay is estimated, leading to the conclusion that the side mode activation is a consequence of the dropouts of the dominant modes. A multimode extension of the Lang-Kobayashi equations is used to model the experimental setup. Numerical simulations also exhibit a time delay between the side-mode activation and the power dropout of the total intensity.
\end{abstract}

Keywords: semiconductor laser, multimode dynamics, low-frequency fluctuations

\section{INTRODUCTION}

In general, the study of the dynamics of the total output power emitted by a multimode semiconductor laser is a complex issue, due basically to the nontrivial interaction between the different modes. From a technological point of view, the use of multimode semiconductor lasers is widespread, due to their low cost and to the fact that in many situations the behavior of their total emitted intensity is qualitatively similar to that of single-mode lasers. On the other hand, some advantages can be extracted from having the power of the laser distributed in various longitudinal modes, like using the different modes for signal multiplexing. ${ }^{1}$ When an external mirror is added to the laser, the system easily exhibits complex dynamical phenomena, such as coherence collapse ${ }^{2}$ (CC) and low-frequency fluctuations ${ }^{3}$ (LFF). In particular, at the regime of low-frequency fluctuations, the output power of the laser drops at irregular times in time scales much longer ( tens of nanoseconds) than the characteristic times of the system dynamics ( $\sim$ tens of picoseconds). This dynamical behavior has been experimentally observed not only in single-mode, but also in multimode semiconductor lasers. ${ }^{4}$ Despite the total output behaves in a similar way in both cases, the modal power in the multimode case shows a nontrivial interaction between longitudinal modes. ${ }^{5,6}$ From the theoretical point of view, the Lang-Kobayashi (LK) model $^{7}$ have been widely used to describe the dynamics of a single-mode semiconductor laser with optical feedback, ${ }^{8}$ with good success in describing both LFF and CC dynamics. The LK equations describe the

E-mail addresses:

J.M.B. (to whom correspondence should be sent): javier.martin-buldu@upc.es

J.G.O.: jordi.g.ojalvo@upc.es

C.R.M.: claudio@imedea.uib.es 
evolution of the complex electric field of the laser and its excess carrier number. Several multimode extensions of this model ${ }^{9-12}$ have been successful in describing qualitatively different experimental observations of mode interactions in multimode semiconductor lasers with optical feedback. ${ }^{5,13}$

When the optical feedback affecting a multimode semiconductor laser is frequency selective (which can be accomplished, e.g., by replacing the external mirror by a diffraction grating), the dropouts of the total output intensity are accompanied by sudden activations of certain longitudinal modes, ${ }^{5,14}$ located at one side of the main mode in the laser spectrum. This side-mode activation has been heuristically interpreted in that frequencyselective case as the cause of the dropouts. ${ }^{5}$ Two different interpretations based on particular multimode extensions of the Lang-Kobayashi model have been proposed. ${ }^{15,16}$ In the present work we study, numerically and experimentally, the relationship between the side-mode activation and the total intensity dropouts in the more general case of a multimode semiconductor laser with global (i.e., non-frequency-selective) optical feedback. We observe and quantify a delay between the dropouts in the main mode of the laser, which dominates the dynamics of the total intensity, and the side-mode activation. Experimental observations confirm the numerical predictions obtained from a multimode LK model. Both experimental and numerical results show that the side-mode activation occurs after the corresponding intensity drop, allowing us to conclude that this activation cannot in this case be the cause, but rather the effect, of the intensity dropout.

\section{MODELING}

\subsection{Multimode Lang-Kobayashi model}

There are different approaches to generalize the standard single-mode Lang-Kobayashi model ${ }^{7}$ in order to describe the behavior of different longitudinal modes of the laser in the presence of feedback. While all of them represent separately the complex envelopes of the electric fields corresponding to the different modes, some of them also distinguish between different carrier densities for the different modes ${ }^{10}$ (in a spirit close to the Tang-Statz-deMars model ${ }^{17}$ ), whereas many others consider that carriers are shared by all modes, ${ }^{9,11,12}$ thus neglecting spatial-hole-burning effects. Some of these models consider mode interaction through various processes, such as self- and cross-saturation, ${ }^{9,12}$ whereas some others introduce a mode-dependent gain. ${ }^{11}$ In what follows, we will make use of the latter type of generalized LK model, consisting of a set of equations for the individual complex amplitudes of the electric fields $E_{m}(t)$ of each mode $m$, and a single equation for the total excess carrier number $N(t)$ of the laser:

$$
\begin{aligned}
& \frac{d E_{m}}{d t}=\frac{1}{2}(1+i \alpha)\left(G_{m}(N)-\gamma_{m}\right) E_{m}(t)+\frac{\kappa}{\tau_{L}} E_{m}(t-\tau) \exp \left(-i \omega_{0 m} \tau\right)+F_{m}(t) \\
& \frac{d N}{d t}=\frac{I}{e}-\frac{N}{\tau_{s}}-\sum_{j=-M}^{M} G_{m}(N)\left|E_{m}\right|^{2}
\end{aligned}
$$

where $m=-M \ldots M$, with $m=0$ corresponding to the mode located at the maximum of the gain curve of the solitary laser. The electric field amplitudes $E_{m}(t)$ are normalized so that $P_{m}(t)=\left|E_{m}(t)\right|^{2}$ measures the photon number in the $m$-th mode. $\alpha$ is the linewidth enhancement factor, $\gamma_{m}$ the mode-dependent cavity loss, and $\tau_{L}$ the round-trip time inside the diode cavity, assumed independent for all modes. $F_{m}(t)$ is a Langevin noise force that accounts for spontaneous emission noise, with a correlation given by $\left\langle F_{m}^{*}(t) F_{n}\left(t^{\prime}\right)\right\rangle=R_{s p} \delta_{m n} \delta\left(t-t^{\prime}\right)$, where $R_{s p}$ is the spontaneous emission rate. In the equation for the carrier density, $\tau_{s}$ is the lifetime of the electron-hole pairs, $I$ is the injection current, and $e$ is the magnitude of the electron charge.

The feedback parameters, namely the feedback level $\kappa$ and the round-trip time of the external cavity $\tau$ are taken to be independent of the mode (in the case of $\kappa$, this independence corresponds to the case of a global feedback). The phase shift $\omega_{0 m} \tau$ appearing in the feedback term is due to the external-cavity roundtrip, with $\omega_{0 m}$ representing the nominal frequency of the $m$-th mode, i.e. $\omega_{0 m}=\omega_{c}+m \Delta \omega_{L}$, where $\omega_{c}$ is the frequency of the gain peak of the solitary laser and $\Delta \omega_{L}$ is the longitudinal mode spacing. 
The mode-dependent gain coefficient $G_{m}$ appearing in the electric field equation (1) is assumed to have a parabolic frequency profile

$$
G_{m}(N)=G_{c}\left(N-N_{0}\right)\left[1-\left(\frac{\omega_{m}-\omega_{\text {peak }}(N)}{\Delta \omega_{g}}\right)^{2}\right],
$$

where $G_{c}$ is the differential gain coefficient at the peak gain of the solitary laser, $N_{0}$ is the carrier number at transparency, $\Delta \omega_{g}$ is the gain width of the laser material, and $\omega_{m}$ is the instantaneous frequency of the $m$-th mode, given by

$$
\omega_{m}(t)=\omega_{0 m}+\frac{d \phi_{m}(t)}{d t} .
$$

In this expression, $\phi_{m}(t)$ is the phase of the slowly varying complex electric field of the $m$-th mode. On the other hand, the center of the parabolic profile (3) occurs at a peak frequency $\omega_{\text {peak }}$ that shifts with the carrier population as ${ }^{18}$

$$
\omega_{\text {peak }}(N)=\omega_{c}+\omega_{N}\left(N-N_{t h}\right)
$$

where $\omega_{N}$ is a constant and $N_{t h}$ is the carrier number at the laser threshold.

In our calculations, we assume nine active optical modes (i.e. $M=4$ ), and consider that $\gamma_{m}$ is mode independent. In this approximation, the spacing between the modes of the solitary laser is given by $\Delta \omega_{L}=2 \pi / \tau_{L}$. We use typical values for the diode laser parameters: $\alpha=4, \tau_{s}=2 \mathrm{~ns}, \tau_{L}=8.3 \mathrm{ps}, \gamma_{m}=5 \times 10^{11} \mathrm{~s}^{-1}$, $G_{c}=4 \times 10^{3} \mathrm{~s}^{-1}, N_{0}=1.1 \times 10^{8}, R_{s p}=5 \times 10^{11} \mathrm{~s}^{-1}$, and $\Delta \omega_{g}=2 \pi \times 2.82 \mathrm{THz}$. Finally, we choose $\omega_{c} \tau=0 \bmod 2 \pi$, so that the feedback phase is $\omega_{0 m} \tau=m \Delta \omega_{L} \tau(\bmod 2 \pi)$, i.e. different for every mode.

\subsection{Side-mode activation}

The LFF regime can be observed in a large range of feedback parameters, whenever the laser is pumped close to its solitary threshold. In the following, we choose $\kappa=7.5 \times 10^{-2}, \tau=4 \mathrm{~ns}$, and $I=1.015 \times I_{t h}$. For these parameters, the total intensity emitted by the laser drops at irregular times, but when we separately observe each longitudinal mode different dynamical behaviors appear. The central modes of the laser with feedback, which are surrounding the mode with largest gain (i.e. the one closer to $\omega_{\text {peak }}, m=-2$ in this case), fall at once, and as a consequence the total intensity exhibits a dropout. But almost at the same time, some longitudinal side modes (closer to the peak frequency $\omega_{c}$ of the solitary laser, i.e. $m=0$ ), that were inactive, jump up when a dropout of the central modes occurs. Figure 1 displays the intensity evolution of the main mode of the laser with feedback, together with that of a side mode exhibiting a jump up. To model the limited bandwidth of the photodetectors used in the experiment, the time traces of the modal intensities and of the total output of the laser have been averaged over a sliding time window of $4 \mathrm{~ns}$.

A closer inspection of Fig. 1 shows that the appearance of the side-mode activations is delayed in relation to the intensity drops of the main mode of the laser with feedback. Figure 2 zooms into one of the LFF events for the two time traces shown in Fig. 1, where the trace exhibiting the dropout has been inverted in order to allow a better comparison with the activation event. The MM drops clearly before the SM jumps. The delay can be estimated to be in the order of the carrier lifetime $\left(\tau_{s}=2 \mathrm{~ns}\right)$, a fact that suggests that the activation is a direct consequence of the loss of power of the main modes (and the consequent shift of the peak frequency to the solitary-laser value ${ }^{19}$ ). These results are observed to be consistent for different dropout events. Statistical determination of the delay time from numerical results of the multimode LK model presented here is still in progress. ${ }^{19}$

\section{EXPERIMENTAL RESULTS}

\subsection{Experimental setup}

In order to confirm the numerical results obtained in the previous section, we implement the experimental setup shown in Fig. 3. The laser chosen in the experiments is an AlGaInP semiconductor laser (Roithner RLT6505G), commonly used in CD devices. It is a multimode laser, with a nominal wavelength of $\sim 650 \mathrm{~nm}$ and a threshold 


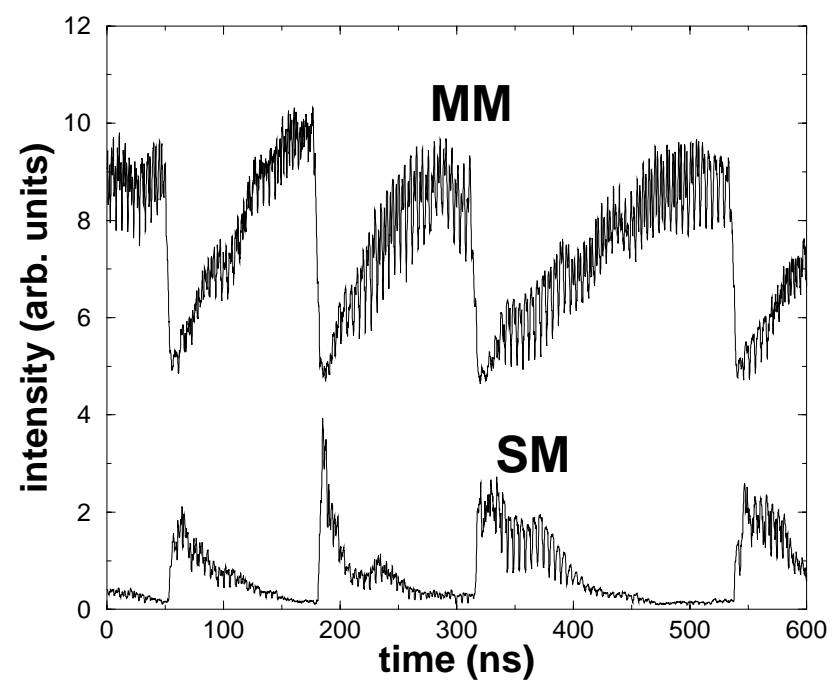

Figure 1. Time series of the intensity of the main mode of the laser with feedback (MM) compared with that of a side mode (SM), obtained from numerical simulations of the multimode LK model described in the text. It can be observed how an activation of the SM is related with a dropout of the MM intensity.

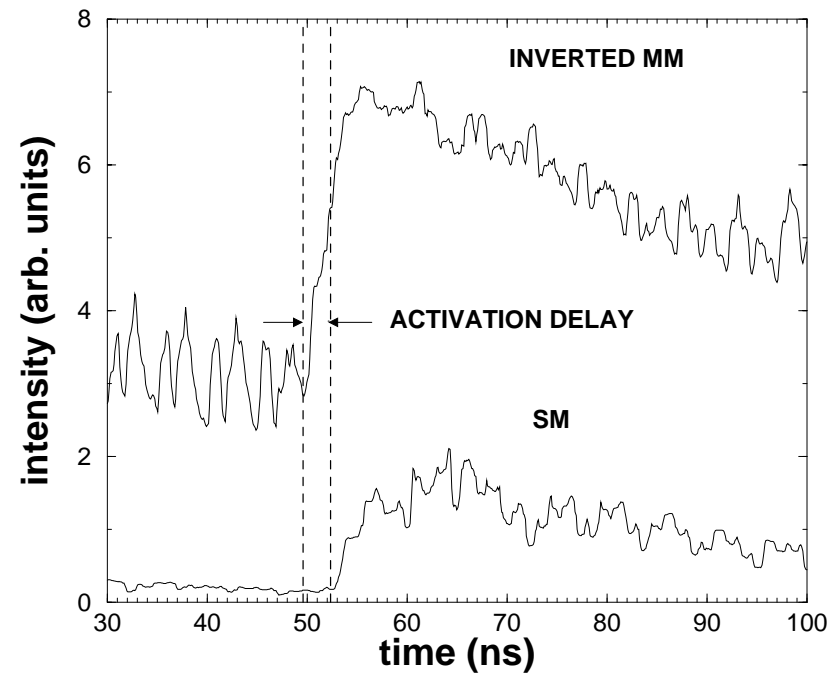

Figure 2. Inverted numerical time series exhibiting one MM dropout compared with the corresponding activation of the intensity of a SM. A delay in the SM activation can be observed.

current of $20.1 \mathrm{~mA}$. An antireflection-coated laser-diode objective is used to collimate the laser output. To introduce an external cavity, we place an external mirror at $L=60 \mathrm{~cm}$ of the laser, which corresponds to a feedback time of $\tau=2 L / c=4 \mathrm{~ns}$. The light reinjected by the mirror produces a threshold reduction of $9.4 \%$ with respect to the solitary laser threshold. The laser intensity is split into two branches, in order to allow the simultaneous monitoring of the total output and of a precise wavelength. The first branch receives part of the total intensity of the laser, which is detected by a fast photodiode and sent to a $500 \mathrm{MHz}$-bandwidth HP 54720D digital oscilloscope. The second branch selects the wavelength to be compared with the total intensity with a $1 / 8 \mathrm{~m}$ CVI monochromator with a resolution better than $0.2 \mathrm{~nm}$. The output of the monochromator, which has lost a considerable amount of power due to the mode selection process, is enhanced by a Hamamatsu PS325 
photomultiplier. The signal received from the photomultiplier is also sent to the oscilloscope. The laser diode is mounted in a TE-cooled mount which allows good control of the temperature and of the injection current of the laser with accuracies of $\pm 0.01{ }^{\circ} \mathrm{C}$ and $\pm 0.1 \mathrm{~mA}$, respectively. We set the injection current to 1.09 times the solitary laser threshold, keeping its temperature at $24.00 \pm 0.01{ }^{\circ} \mathrm{C}$.

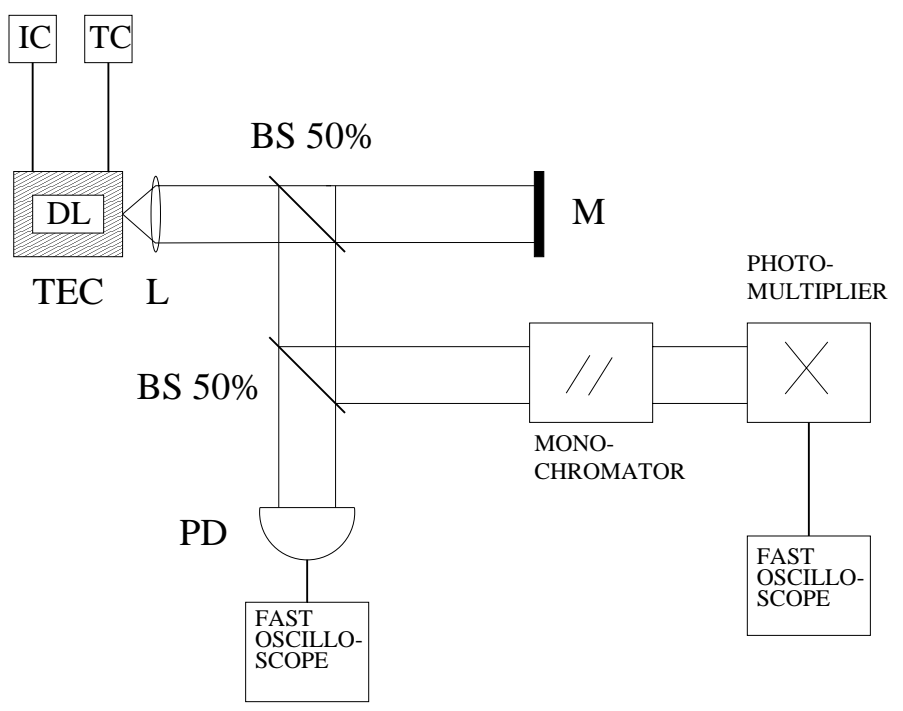

Figure 3. Experimental setup: DL, diode laser; BS, beam splitter; M, external mirror; TEC, laser diode mount; PD, photodiode; IC, intensity controller; TC, temperature controller.

Before introducing the feedback, we analyze the time-averaged optical spectrum of the solitary laser by scanning the relevant wavelength range with the monochromator. The result is shown in Fig. 4, from which one can conclude (with a limited resolution) that the laser operates at least in ten longitudinal modes, with its maximum located at a wavelength $\approx 658.4 \mathrm{~nm}$ and a total $\mathrm{FWHM}$ of $\approx 0.9 \mathrm{~nm}$. When the external mirror is added to the system, the spectrum broadens up to a FWHM of $\approx 1.3 \mathrm{~nm}$, and its maximum shifts $\approx 0.5 \mathrm{~nm}$ towards higher wavelengths.

\subsection{Side-mode activation}

By adjusting the feedback to the parameters mentioned above, we force the laser to emit in the LFF regime, observing intensity dropouts of the total output. In this regime, we have compared in Fig. 5 the dynamical behavior of the main mode (MM) of the laser with feedback (i.e., for the maximum at the corresponding curve in Fig. 4), with that of the total intensity (traces a-b). It can be observed how the dynamical response is qualitatively the same in the two cases, consisting in dropouts in both measures. The behavior of longitudinal side modes (SM) close to the main mode of the solitary laser is also compared with that of the total intensity (traces c-d), exhibiting a different dynamical behavior. When a dropout appears at the total intensity, a sudden activation of the side modes is observed. Note that the intensity recovery in the case of the MM is much slower than the recovery of the total intensity, a fact that has been already reported in the literature. ${ }^{5,6}$ Another feature observed in the experimental results is that the activation is not symmetric, being practically absent on the high-wavelength part of the spectrum (the wavelength range where the phenomenon is basically observed is indicated in Fig. 4). The side-mode activation has been observed in different multimode lasers of the same line (Roithner RLT6505G), and also in other types of lasers, including nearly-single-mode lasers (Sony SLD1132VS and SLD1137VS).

Figures 5(a) and (b) display a delay between the dropout of the total intensity and the dropout of the MM. This delay, estimated in $\approx 20 \mathrm{~ns}$, is artificial and due to the response time of the photomultiplier, which is only used in the mode-selecting branch. This delay can be also observed in Figs. 5 (c) and (d), in this case between 


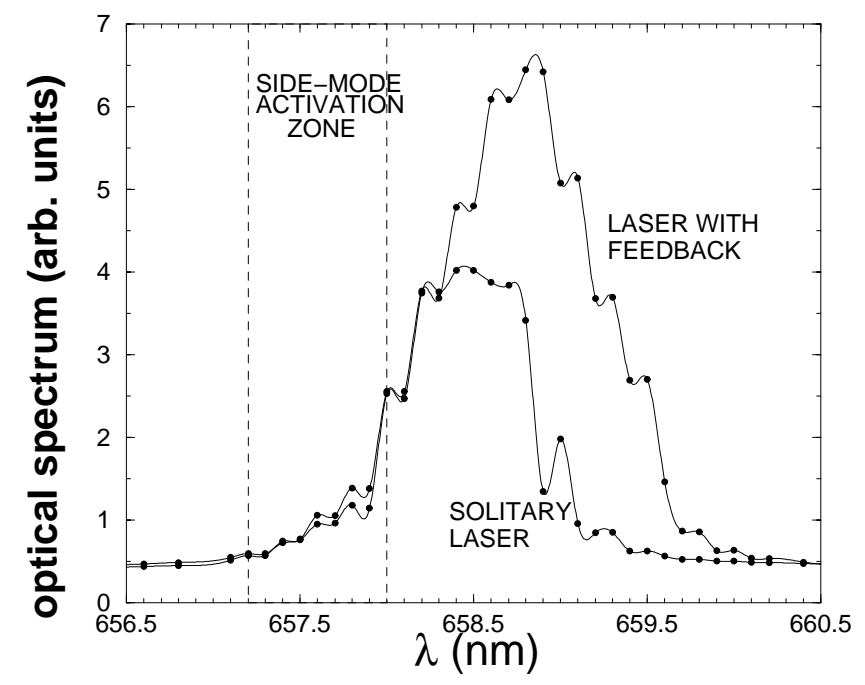

Figure 4. Optical spectrum of our multimode semiconductor laser with and without feedback. It can be seen how feedback enhances the higher wavelength limit of the gain curve.

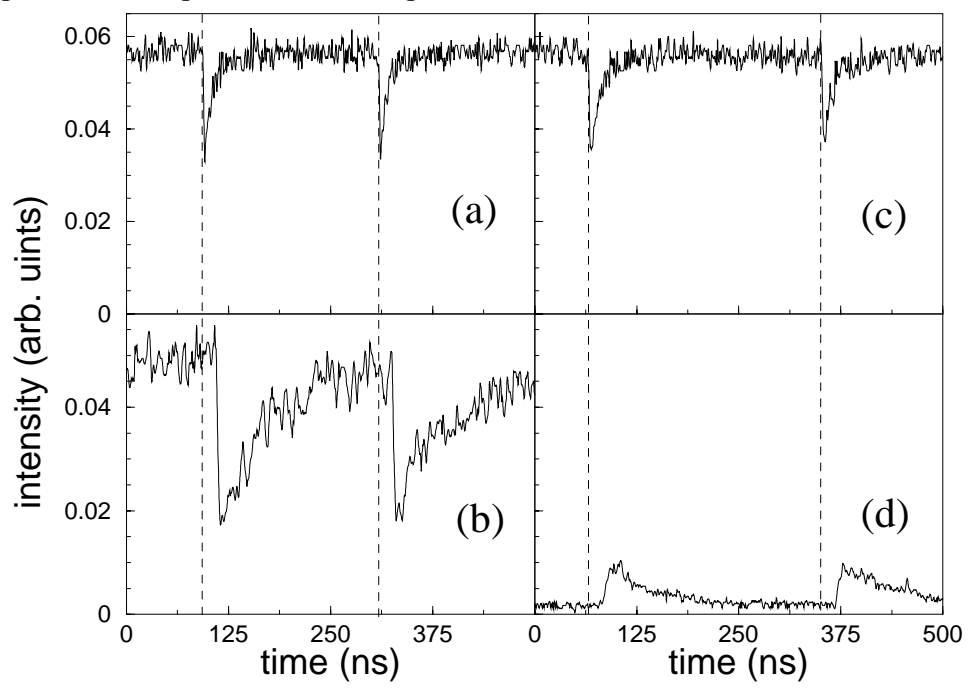

Figure 5. Experimental time traces showing the modal structure of a dropout. Total intensity evolution (a, c) compared to that of the main mode of the laser with feedback (b) and a side mode that activates with LFF (d). Although traces $(\mathrm{a}, \mathrm{b})$ and $(\mathrm{c}, \mathrm{d})$ have been acquired simultaneously, a delay from the total output can be observed, due to the response time of the photomultiplier.

the dropout of the total intensity and the side-mode activation. However, a closer inspection of these last results reveal that the delay is in this case a combination of the response time of the photomultiplier plus the delay of the side-mode activations with respect to the dropouts of the main mode (MM), which was observed in the numerical simulations, as described in Section 2. A more detailed analysis of these results is shown in Fig. 6, where two pairs of total-output/MM and total-output/SM time series are presented together, conveniently shifted so that the total-output dropouts coincide in time. Under these conditions, one can observe both the electronic delay introduced by the photomultiplier and the delay between a MM dropout and a SM activation. In order to separate the delay introduced by the photomultiplier we compare the dropout of the MM with the 


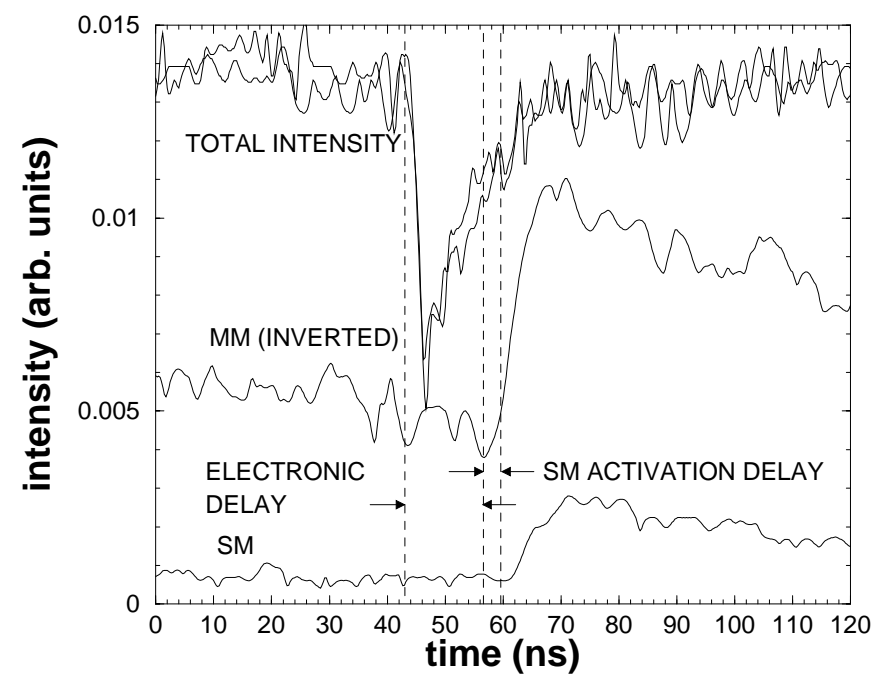

Figure 6. Time traces of the total intensity (two of them), the MM dropout (inverted) and the SM activation. The traces are simultaneous in pairs (such as in Fig. 5).

dropout of the total intensity and consider this time as the electronic delay time. To statistically obtain the electronic delay we average the total output and the MM time traces in series of 40 dropouts, with the aim of averaging out the fluctuations before a dropout event. Once the series are averaged, we observe that both intensities fall with the same slope, which allows us to measure the time between both dropouts. ${ }^{20}$ A total of 3000 dropout events are statistically studied, leading to an electronic delay time of $15.6 \pm 0.81 \mathrm{~ns}$. We next proceed to analyze the delay between the total-output dropout and the SM activation in the same manner, measuring a delay time of $17.10 \pm 0.71 \mathrm{~ns}$. Removing the electronic delay from the total delay obtained for the SM activation we obtain a remaining delay of $1.5 \pm 1.1 \mathrm{~ns}$ between the MM dropout and the SM activation. Figure 7 presents the distribution of the delay times of the MM from the total output (electronic delay), and that of the SM activation from the total output (electronic plus activation delay). As it has been observed in the numerical simulations, the SM activation occurs after the dropout of the MM, leading to the conclusion that it is an effect, and not the cause, of the MM dropout.

\section{CONCLUSIONS}

In conclusion, we have numerically and experimentally studied the mode interaction of a semiconductor laser with non-selective feedback in the low-frequency-fluctuation regime. The power dropouts are accompanied by activations of side modes with lower wavelengths. We interpret this asymmetric activation to be the result of the shift of the gain curve towards higher wavelengths in the presence of feedback. We have compared the occurrence times of these activations with that of the power dropouts of the main mode of the laser with feedback, observing a delay between the latter and the former. We have quantified this delay from both our numerical and experimental results, obtaining a value in the order of the carrier lifetime of this kind of lasers. Hence, assuming that the dropout in the main mode is simultaneous to that in the total intensity, we reach the conclusion that the side-mode activation is a consequence of the dropout of the total intensity. Considering the good agreement between the experimental observations and the numerical simulations, we can also conclude that a multimode extension of the Lang-Kobayashi model (including a parabolic gain profile and a carrier-dependent peak frequency) describes correctly this phenomenology. 


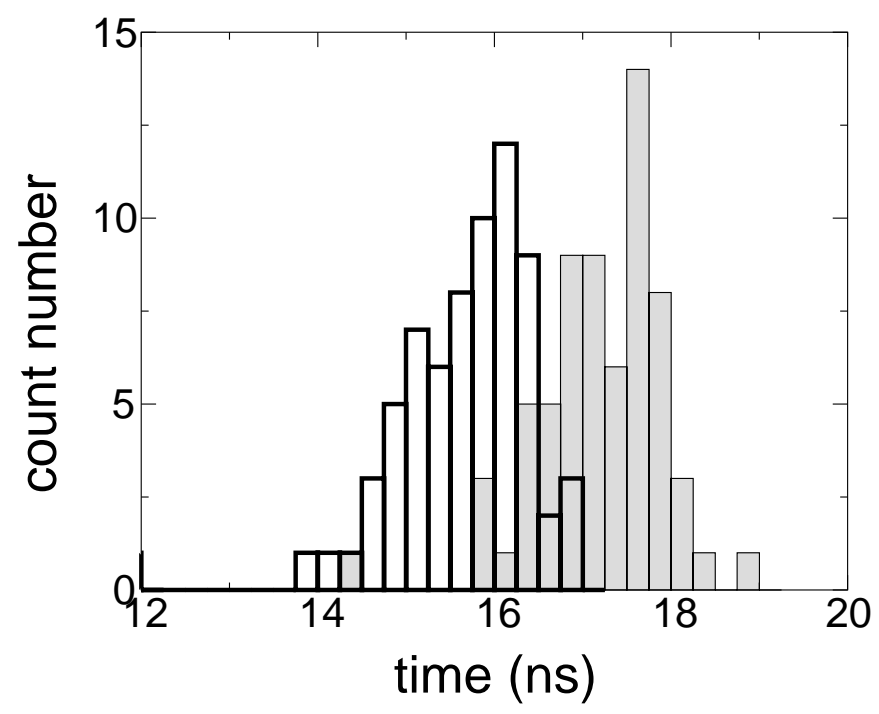

Figure 7. Distribution of occurrence times of both the main-mode dropouts (white bars) and the side-mode activations (grey bars).

\section{ACKNOWLEDGMENTS}

We thank J. Martorell and R. Vilaseca for lending us part of the experimental setup. We acknowledge financial support from Ministerio de Ciencia y Tecnologia (Spain), under projects PB98-0935, BFM2000-0624 and BFM2001-2159, from the EU IST network OCCULT, and from the Generalitat de Catalunya, under project 1999SGR-00147. FR is supported by the Inter-University Attraction Pole program (IAP IV/07) of the Belgian government.

\section{REFERENCES}

1. J.K. White and J.V. Moloney, Phys. Rev. A 59, 2422, 1999.

2. D. Lenstra, B. Verbeek, and A.J. de Boef, IEEE J. Quantum Electron. 21, 764, 1985.

3. Ch. Risch and C. Voumard, J. Appl. Phys. 48, 2083, 1977.

4. D. Yu, I. Wallace, R.G. Harrison and A. Gavrielides, Opt. Comm. 195, 249, 2001.

5. G. Huyet, S. Balle, M. Giudici, C. Green, G. Giacomelli, and J. R. Tredicce, Opt. Comm. 149, 341, 1998.

6. I. Wallace, D. Yu, R. G. Harrison and A. Gavrielides, J. Opt. B 2, 447, 2000.

7. R. Lang and K. Kobayashi, IEEE J. Quantum Electron. 16, 347, 1980.

8. T. Sano, Phys. Rev. A 50, 2719, 1994; I. Fischer, G.H.M. van Tartwijk, A.M. Levine, W. Elsäßer, E. Göbel, and D. Lenstra, Phys. Rev. Lett. 76, 220, 1996; C. Masoller and N.B. Abraham, Phys. Rev. A 57, 1313, 1998; T. Heil, I. Fischer, W. Elsäßer, J. Mulet, and C.R. Mirasso, Opt. Lett. 24, 1275, 1999.

9. A.T. Ryan, G.P. Agrawal, G. R. Gray, and E. C. Gage, IEEE J. Quantum Electron. 30, 668, 1994.

10. E.A. Viktorov and P. Mandel, Phys. Rev. Lett. 85, 3157, 2000; T.W. Carr, D. Pieroux, and P. Mandel, Phys. Rev. A 63, 033817, 2001.

11. F. Rogister, P. Mégret, O. Deparis and M. Blondel, Phys. Rev. A 62, 061803(R), 2001.

12. D.W. Sukow, T. Heil, I. Fischer, A. Gavrielides, A. Hohl-AbiChedid, and W. Elsäßer, Phys. Rev. A 60, $667,1999$.

13. G. Vaschenko, M. Giudici, J.J. Rocca, C.S. Menoni, J.R. Tredicce, and S. Balle, Phys. Rev. Lett. 81, 5536, 1998. 
14. M. Giudici, L. Giuggioli, C. Green, and J.R. Tredicce, Chaos, Solitons and Fractals 10, 811, 1999.

15. E.A. Viktorov and P. Mandel, Opt. Lett. 25, 1576, 2000.

16. F. Rogister, M. Sciamanna, O. Deparis, P. Mégret and M. Blondel, Phys. Rev. A 65 015602, 2002.

17. C.L. Tang, H. Statz, G. deMars, J. Appl. Phys. 34, 2289, 1963.

18. J. Mork, B. Tromborg and P.L. Christiansen, IEEE J. Quantum Electron. 24, 123, 1988.

19. J.M. Buldú, F. Rogister, J. Trull, C. Serrat, M.C. Torrent, C.R. Mirasso, and J. García-Ojalvo, in preparation, 2001.

20. J.M. Buldú, J. Trull, M.C. Torrent, J. García-Ojalvo, and C.R. Mirasso, J. Opt. B, to appear, 2002. 